\title{
ARTICLE
}

\section{Response measurement of various neutron dose equivalent monitors in 134-387 MeV neutron fields}

\author{
Yoshihiro Nakane $^{\mathrm{a} *}$, Masayuki Hagiwara ${ }^{\mathrm{b}}$, Yosuke Iwamoto ${ }^{\mathrm{a}}$, Hiroshi Iwase ${ }^{\mathrm{b}}$, Daiki Satoh ${ }^{\mathrm{a}}$, Tatsuhiko Sato ${ }^{\mathrm{a}}$, \\ Hiroshi Yashima ${ }^{c}$, Tetsuro Matsumoto ${ }^{\mathrm{d}}$, Akihiko Masuda ${ }^{\mathrm{d}}$, Tomoya Nunomiya ${ }^{\mathrm{e}}$, Yukio Sakamoto ${ }^{\mathrm{f}}$, \\ Hiroshi Nakashima ${ }^{\mathrm{a}}$, Atsushi Tamii ${ }^{\mathrm{g}}$, Kichiji Hatanaka ${ }^{\mathrm{g}}$ and Takashi Nakamura ${ }^{\mathrm{h}}$ \\ ${ }^{a}$ Japan Atomic Energy Agency, 2-4 Shirakata, Tokai-mura, Naka-gun, Ibaraki-ken, 319-1195, Japan; ${ }^{b}$ High Energy Accelerator \\ Research Organization (KEK), 1-1 Oho, Tsukuba-shi, Ibaraki-ken, 305-0801, Japan; ${ }^{c}$ Research Reactor Institute, Kyoto \\ University, 2 Asashiro-Nishi, Kumatori-cho, Sennan-gun, Osaka, 590-0494, Japan; ${ }^{d}$ National Institute of Advanced Industrial \\ Science and Technology, 1-1-1 Higashi, Tsukuba-shi, Ibaraki-ken, 305-8561, Japan; ${ }^{e}$ Fuji Electric Co., Ltd., 1 Fuji-machi, \\ Hino-shi, Tokyo, 191-8502, Japan; ${ }^{f}$ ATOX Co., Ltd., 1201 Takada, Kashiwa-shi, Chiba, 277-0861, Japan; ${ }^{g}$ Research center for \\ Nuclear Physics, 10-1 Mihogaoka, Ibaraki-shi, Osaka University, Osaka, 567-0047, Japan; ${ }^{h}$ Tohoku University, 6-6 Aoba, \\ Aramaki, Aoba-ku, Sendai, 980-8578, Japan
}

In order to verify the accuracy of dose monitors for the measurements of high-energy neutrons, energy responses of seven neutron dose equivalent monitors, four kinds of wide-energy range monitors applied to high-energy neutron fields above $20 \mathrm{MeV}$, and three kinds of conventional type monitors for neutrons up to $20 \mathrm{MeV}$, were measured for 134-, 197-, 244- and 387-MeV quasi-monoenergetic neutron fields. Measured dose rates were compared one another, and with those in the fields obtained from neutron energy spectra and flux-to-dose conversion coefficients considered on design of the monitors. Dose rates measured with the wide-energy range monitors were in agreement or overestimate with evaluated ones, though those measured with the conventional type monitors underestimate. It was found that the wide-energy range monitors used in the present work will give the dose rates with a safety side at several hundred MeV neutron fields, though the conventional type monitors underestimate the dose rate in the fields.

Keywords: high-energy; quasi-monoenergetic neutron; neutron dose; dose equivalent monitors; wide-energy range monitors; energy response

\section{Introduction}

In recent years, a lot of experiments in the field of frontier science were performed with using high energy radiations generated by high intensity accelerators. In Japan, high intensity proton accelerator facility, J-PARC[1], has been constructed for science and physics research as the joint project between the Japan Atomic Energy Agency (JAEA) and the High Energy Accelerator Research Organization (KEK). For the radiation protection in high-energy neutron fields such as found in accelerator facilities, it is important to measure the dose rates caused by wide-energy range neutrons, accurately. Especially, the dose rates caused by neutrons from 20 to several hundred $\mathrm{MeV}$ are important for the radiation protection in the fields because these neutrons dominantly contribute to the dose equivalent in general. In low-energy neutron fields below $20 \mathrm{MeV}$, many kinds of commercial neutron dose equivalent monitors such as AnderssonBraun type [2] dose monitors have been used widely for the measurements of neutron dose rates. The monitors, however, generally underestimate the dose caused by high-energy neutrons above $20 \mathrm{MeV}$ because these monitors are designed for the measurement up to 20 $\mathrm{MeV}$. Therefore, wide-energy range monitors have been developed for the improvement of dose measurements at high-energy neutron fields [3-6].

In order to verify the accuracy of dose monitors for the measurements of high-energy neutrons, energy responses were measured in the present work by seven kinds of neutron dose equivalent monitors for 134-, 197-, 244- and 387-MeV quasi-monoenergetic neutrons generated from the ${ }^{7} \mathrm{Li}(\mathrm{p}, \mathrm{n})$ reactions at the Research Center of Nuclear Physics (RCNP) ring cyclotron of Osaka University [7]. Dose rates measured by the monitors were compared with one another, and with those in the fields obtained from neutron energy spectra and flux-to-dose conversion coefficients.

*Corresponding author. Email: nakane.yoshihiro@jaea.go.jp 


\section{Experiments}

\subsection{Experimental setup}

Experiments were carried out at the neutron time-of-flight (TOF) beam course in the RCNP of Osaka University. Figure 1 shows a schematic view of the experimental arrangement. The experimental room is a tunnel of $100-\mathrm{m}$ in length, 4-m in width and $3.5-\mathrm{m}$ in height. Quasi-monoenergetic source neutrons, the peak energies were 134-, 197-, 244- and 387-MeV, were produced in a $1.0-\mathrm{cm}$ thick Li target bombarded with 137-, 200-, 246- and 389-MeV protons, respectively. Source neutrons emitted in the forward direction reached an experimental room through a 10.0-cm-wide and 12.0-cm-height aperture, and 150-cm-long iron collimator embedded in a concrete wall. The protons penetrating through the target were bent towards a beam dump by the swinger magnet to measure the proton beam intensity with a Faraday cup inside the dump. The monitors were placed on the neutron beam at the position of $35.0 \mathrm{~m}$ from the $\mathrm{Li}$ target except for $387-\mathrm{MeV}$ neutron, $30.0 \mathrm{~m}$ for $387-\mathrm{MeV}$ neutron. At the $35.0 \mathrm{~m}$ position, the size of collimated beam is about $60 \times 70 \mathrm{~cm}^{2}$. Since the beam size is quite small to the room size, contributions of wall-scatter components were estimated to be quite small.

The energy spectra of source neutrons were shown in Figure 2. The spectra have been measured by the TOF methods with three sizes of NE213 liquid organic scintillation detectors $\left(25.4 \times 25.4 \mathrm{~cm}^{2}\right.$ for neutrons above $100 \mathrm{MeV}, 12.7 \times 12.7 \mathrm{~cm}^{2}$ for neutrons from 10 to $100 \mathrm{MeV}$, and $5.08 \times 5.08 \mathrm{~cm}^{2}$ for neutrons below 10 $\mathrm{MeV}$ in diameter and length) [8-9].

\subsection{Neutron monitors}

Table 1 shows the neutron dose equivalent monitors used in the present work. Four kinds of wide-energy range monitors applicable for high-energy neutron field (three kinds of modified Andersson-Braun type monitors with lead or tungsten breeder, and a liquid organic scintillator type monitor with the response functions applied for high-energy neutron dose) were used in the measurements. In addition, three kinds of conventional

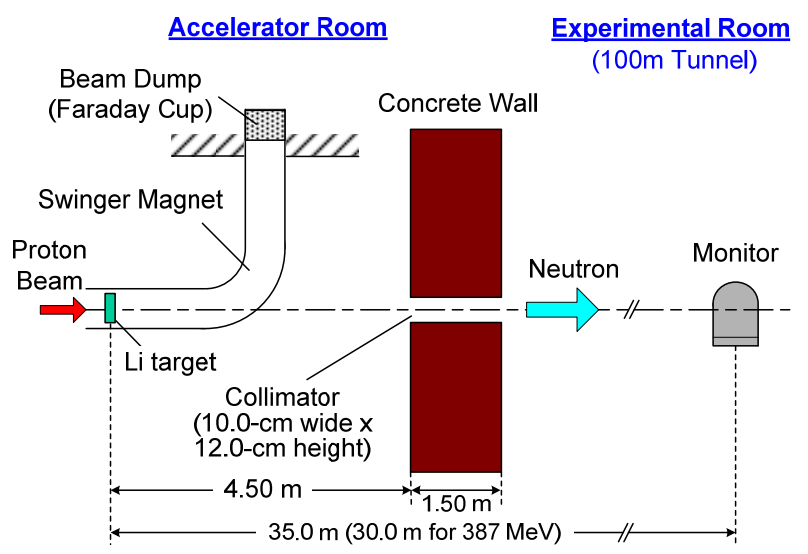

Figure 1. Schematic view of the experimental arrangement at RCNP.

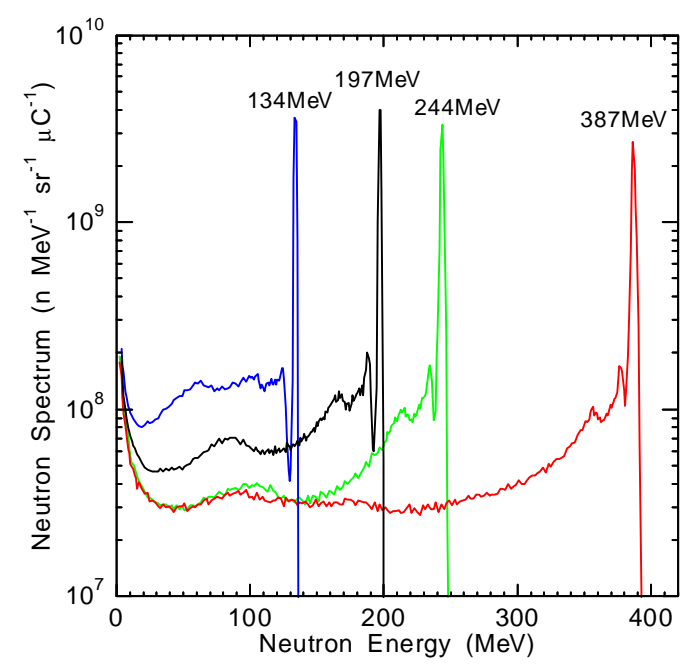

Figure 2. Measured energy spectra of source neutrons.

type monitors (two Andersson-Braun type monitors and a proportional counter type monitor) were also used for comparisons. In the table, the JAEA wide range monitor was developed [6] for applying to neutron monitoring for high-energy proton accelerator facility, J-PARC [1], and products of the monitor have been stationed in the facility for monitoring neutron dose rates. To measure the neutron dose accurately for wide energy

Table 1. Neutron dose equivalent monitors used in the present work.

\begin{tabular}{|c|c|c|c|c|c|c|c|}
\hline \multirow{3}{*}{ Monitor } & \multirow{3}{*}{$\begin{array}{l}\text { Wide- } \\
\text { energy } \\
\text { range }\end{array}$} & \multirow{2}{*}{\multicolumn{2}{|c|}{$\begin{array}{c}\text { Reproduced } \\
\text { dose conversion } \\
\text { coefficients }\end{array}$}} & \multirow{3}{*}{ Detector type } & \multicolumn{3}{|c|}{ Data of Andersson-Braun type } \\
\hline & & & & & \multirow{2}{*}{ Counter } & \multirow{2}{*}{$\begin{array}{l}\text { Breeder for } \\
\text { high-energy } \\
\text { neutron }\end{array}$} & \multirow{2}{*}{ Shape } \\
\hline & & $\leq 20 \mathrm{MeV}$ & $>20 \mathrm{MeV}$ & & & & \\
\hline JAEA wide range & yes & $\mathrm{H}^{*}(10)$ & $E$ & Modified Andersson-Braun & ${ }^{3} \mathrm{He}$ & Lead & Spherical \\
\hline HPI* 6060 & yes & $H^{*}(10)$ & $\mathrm{H}^{*}(10)$ & Modified Andersson-Braun & $\mathrm{BF}_{3}$ & Lead & Cylindrical \\
\hline WENDI & yes & $\mathrm{H}^{*}(10)$ & $\mathrm{H}^{*}(10)$ & Modified Andersson-Braun & ${ }^{3} \mathrm{He}$ & Tungsten & Cylindrical \\
\hline DARWIN & yes & $H^{*}(10)$ & $\mathrm{H}^{*}(10)$ & Liquid organic scintillator & --- & --- & --- \\
\hline Alnor(Studsvik) 2202D & no & $\mathrm{H}^{*}(10)$ & --- & Andersson-Braun & $\mathrm{BF}_{3}$ & none & Cylindrical \\
\hline Fuji Electric NSN1 & no & $H^{*}(10)$ & --- & Andersson-Braun & ${ }^{3} \mathrm{He}$ & none & Spherical \\
\hline Fuji Electric NSN3 & no & $H^{*}(10)$ & --- & Proportional counter & --- & --- & --- \\
\hline
\end{tabular}

* Health Physics instruments 
range, a 1.5-cm-thick lead spherical shell was inserted to a conventional Andersson-Braun type rem monitor between inner and outer polyethylene spherical shells as a breeder for high-energy neutrons, which is similar concept for other modified Andersson-Braun type monitors. The thickness and the position of the lead shell inserted the monitor were determined [6] by neutron transport calculations using MCNP-4B code [10] with LA150 library [11] that the energy response reproduces the flux-to-dose conversion coefficients. For neutron energy below $20 \mathrm{MeV}$, the monitor was designed to reproduce the coefficients for ambient dose equivalent [12-14], $H^{*}(10) / \phi$, same as the other monitors. On the other hand, the responses reproduce the coefficients for effective dose [15], $E / \phi$, for the energy above $20 \mathrm{MeV}$, though the other monitors were designed to reproduce those of ambient dose equivalent, $\mathrm{H}^{*}(10) / \phi$, because the monitor responses do not underestimate dose rates at high-energy neutron fields. For verifying the calculations of energy response, the responses have been measured at neutron fields from thermal to 68-MeV quasi-monoenergetic neutron sources[6]. The calculation responses were in good agreement with measured ones within $15 \%$, and the measured dose rates were in good agreement with evaluated ones within $10 \%$.

\subsection{Evaluations of dose rates in the fields}

\subsubsection{Evaluations of dose rates}

Neutron dose rates in the fields have been evaluated from the measured energy spectra of source neutrons and flux-to-dose conversion coefficients. According to the design concept of the monitors, two kinds of the coefficients, $\mathrm{H}^{*}(10)$ and $E$, were applied for neutron energy above $20 \mathrm{MeV}$ in the evaluation.

\subsubsection{Dose conversion coefficients}

In general, monitors are designed that the energy responses reproduce to the coefficients of $\mathrm{H}^{*}(10)$ as operational quantities. On the other hand, the coefficients for $E$ were applied for the shielding calculations of the facility as protection quantities. Figure 3 shows the comparisons of the coefficients [12-15]. The coefficients for $\mathrm{H}^{*}(10)$ are obviously smaller than those for $E$ for the energy above $100 \mathrm{MeV}$, only $55-60 \%$ of those for $E$ for $200-400 \mathrm{MeV}$, while those are in agreement for the low energy region on the whole. It means that the monitors designed for reproducing the coefficients of $\mathrm{H}^{*}(10)$ have a possibility of underestimating effective dose rate in high-energy neutron fields above $100 \mathrm{MeV}$. The JAEA wide range

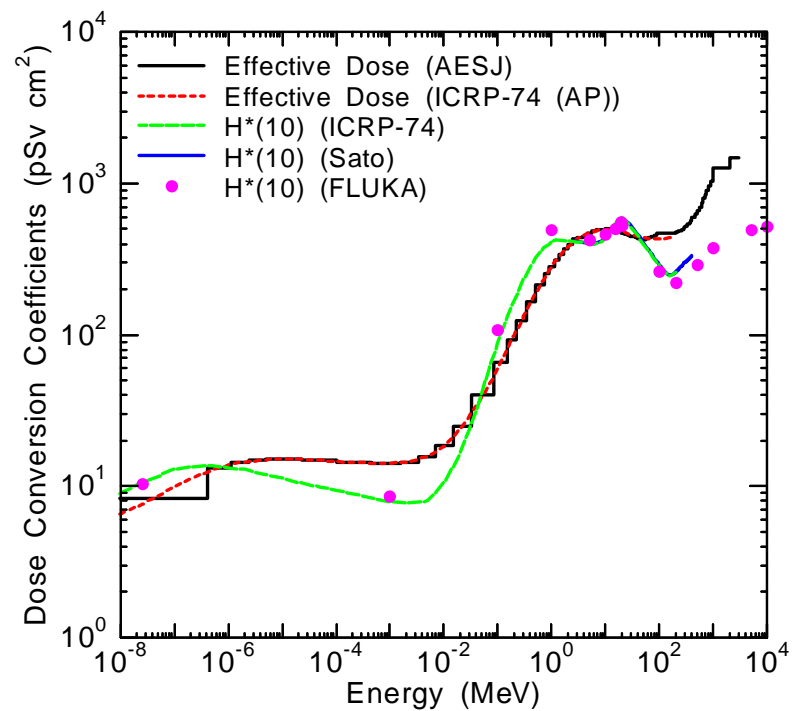

Figure 3. Flux-to-dose conversion coefficients for effective dose and ambient dose equivalent.

monitor was designed to avoid this underestimation. For the comparisons of energy regions, both the coefficients above $1 \mathrm{MeV}$ are several tens of times larger than those below $10 \mathrm{keV}$. This means that the contribution of low-energy neutrons is estimated to be quite smaller in dose evaluation than that of high-energy neutrons.

\subsubsection{Evaluated results of dose rates}

Table 2 shows the evaluated dose rates per proton beam intensity(coulomb) at the monitor irradiation position, $35.0 \mathrm{~m}$ distance from $\mathrm{Li}$ target. The results show that dose rates applied for $\mathrm{H}^{*}(10)+E$ are $44-65 \%$ larger than the those for $\mathrm{H}^{*}(10)+\mathrm{H}^{*}(10)$ in the field because of the differences between $\mathrm{H}^{*}(10)$ and $E$ for the energy above $100 \mathrm{MeV}$ shown in Figure 3.

\section{Results and discussions}

\subsection{Comparison of measured results}

Measured results of monitor responses are listed in Table 3. The results for the $387-\mathrm{MeV}$ source are normalized to those at the position of $35.0 \mathrm{~m}$ from the $\mathrm{Li}$ target. The measured results of all the conventional type monitors are smaller than those of all the wide-energy range monitors. Especially, the results of Alnor 2202D and NSN1 are about an order smaller than those of JAEA wide range.

For the comparisons between wide-energy range

Table 2. Calculated neutron dose rates at the position of $35.0 \mathrm{~m}$ from the Li target.

\begin{tabular}{|c|c|c|c|c|c|c|}
\hline \multirow[t]{2}{*}{ Case of calculation } & \multicolumn{2}{|c|}{$\begin{array}{l}\text { Applied flux-to-dose } \\
\text { conversion coefficients }\end{array}$} & \multicolumn{4}{|c|}{$\begin{array}{l}\text { Calculated dose rates per proton beam intensity }\left(\mathrm{Sv} \mathrm{C} \mathrm{C}^{-1}\right) \\
\text { at the position of } 35.0 \mathrm{~m} \text { from the Li target }\end{array}$} \\
\hline & $\mathrm{En} \leq 20 \mathrm{MeV}$ & $\mathrm{En}>20 \mathrm{MeV}$ & $134 \mathrm{MeV}$ & $197 \mathrm{MeV}$ & $244 \mathrm{MeV}$ & $387 \mathrm{MeV}$ \\
\hline$H^{*}(10)+H^{*}(10)$ & $H^{*}(10) / \phi$ & $H^{*}(10) / \phi$ & 0.665 & 0.592 & 0.545 & 0.699 \\
\hline$H^{*}(10)+E$ & $H^{*}(10) / \phi$ & $E / \phi$ & 0.961 & 0.952 & 0.885 & 1.154 \\
\hline
\end{tabular}


Table 3. Summary of measured responses and the ratios of evaluated dose rates.

\begin{tabular}{|c|c|c|c|c|c|c|c|c|}
\hline \multirow[t]{2}{*}{ Monitor } & \multicolumn{4}{|c|}{$\begin{array}{l}\text { Measured dose rates per beam intensity }\left(\mathrm{Sv} \mathrm{C}^{-1}\right) \\
\text { at the position of } 35.0 \mathrm{~m} \text { from the Li target }\end{array}$} & \multicolumn{4}{|c|}{$\begin{array}{l}\text { Ratio of measured and evaluated dose rates } \\
\text { applied for } H^{*}(10)+H^{*}(10) \text { (for } H^{*}(10)+E \text { ) }\end{array}$} \\
\hline & $134 \mathrm{MeV}$ & $197 \mathrm{MeV}$ & $244 \mathrm{MeV}$ & $387 \mathrm{MeV}^{*}$ & $134 \mathrm{MeV}$ & $197 \mathrm{MeV}$ & $244 \mathrm{MeV}$ & $387 \mathrm{MeV}^{*}$ \\
\hline JAEA wide range & 1.11 & 1.17 & 1.32 & 1.74 & (1.15) & (1.23) & $(1.49)$ & (1.51) \\
\hline HPI 6060 & --- & --- & 0.621 & 0.855 & --- & --- & 1.14 & 1.22 \\
\hline DARWIN & --- & --- & 0.725 & 0.725 & --- & --- & 1.33 & 1.04 \\
\hline WENDI & --- & --- & 0.737 & 1.115 & --- & --- & 1.35 & 1.60 \\
\hline Alnor 2202D & --- & --- & 0.137 & 0.163 & --- & --- & 0.252 & 0.233 \\
\hline Fuji NSN1 & --- & --- & 0.116 & --- & --- & --- & 0.212 & --- \\
\hline Fuji NSN3 & 0.513 & 0.414 & 0.364 & --- & 0.771 & 0.699 & 0.668 & --- \\
\hline
\end{tabular}

* Results of $387 \mathrm{MeV}$ were normalized to those at the position of $35.0 \mathrm{~m}$ from the Li target.

monitors, the results of HPI 6060, DARWIN and WENDI are in agreements one another. The results of JAEA wide range was 1.5-2.4 times larger than those of others. It was because the differences of reproducing the coefficients on the design. As shown in figure 3, the coefficients for $E$ are about twice larger than those for $\mathrm{H}^{*}(10)$ for the energy above $200 \mathrm{MeV}$.

For the comparisons between conventional type monitors, measured result of Alnor 2202D is in agreement with that of NSN1 for the $244-\mathrm{MeV}$ source. On the other hand, the result of NSN3 is about 3 times larger than those of Alnor 2202D and NSN1, and close to the results of wide-energy range monitors. This agreement and disagreement is ascribed to be the difference of detector types, as shown in Table 1.

\subsection{Comparison of measured to calculated results}

Measured dose rates were compared with calculated ones obtained from the coefficients considered on design. The ratios of measured and evaluated dose rates are listed in Table 3, and shown in Figure 4. The results of all the monitors except JAEA wide range were compared with those for $\mathrm{H}^{*}(10)$, though those of JAEA wide range were compared with those for $\mathrm{H}^{*}(10)+E$. The measured results with JAEA wide range were in agreement with evaluated ones within $23 \%$ for the 134 and the 197-MeV sources. Although the results of

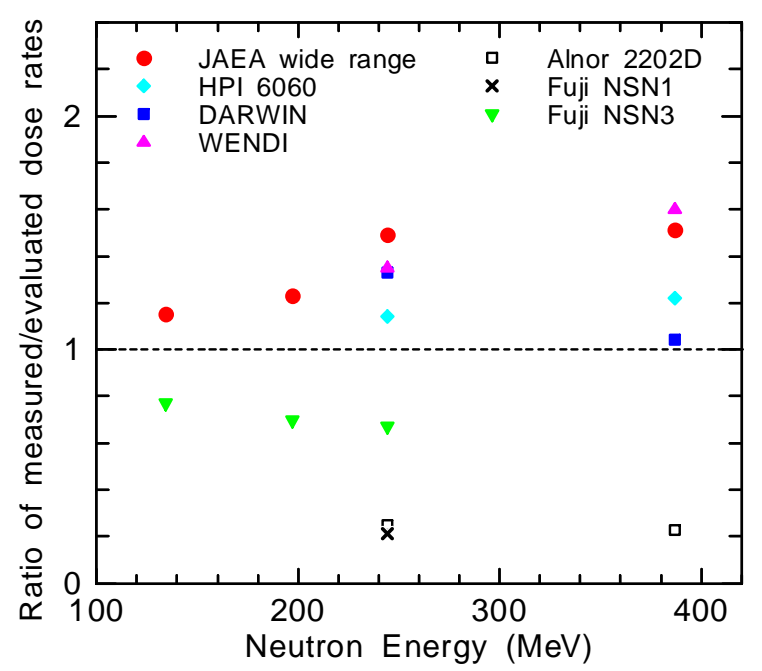

Figure 4. Ratios of measured and evaluated dose rates.
NSN3 were in agreement with evaluated ones within $31 \%$, it was a problem for dose monitor to underestimate dose rate at the fields. For the 244- and the 387-MeV sources, the results of JAEA wide range and WENDI tend to overestimate the evaluated ones, though the results of HPI and DARWIN were in agreement on the whole with the evaluated ones. On the other hand, the results of all the conventional type monitors underestimate the evaluated ones. Especially, the results of Alnor 2202D and NSN1 (conventional Andersson-Braun type monitors) are remarkably underestimate, only $21-25 \%$ of the evaluated ones.

\section{Conclusion}

The energy responses for seven kinds of neutron dose equivalent monitors were measured for $134-387 \mathrm{MeV}$ quasi-monoenergetic neutrons. Dose rates measured with four wide-energy range monitors are in agreement or overestimate with evaluated ones in the fields. On the other hand, all the conventional type monitors underestimate the evaluated ones, especially the results of conventional Andersson-Braun type monitors show only $21-25 \%$. It is concluded that the wide-energy range monitors used in the present work will give the dose rates with a safety side at several hundred $\mathrm{MeV}$ neutron fields, though the conventional type monitors underestimate the dose rate in the fields.

\section{References}

[1] S. Tanaka, High intensity proton accelerator project in Japan (J-PARC), Radiat. Protec. Dosim. 115 (2005), pp. 33-43.

[2] I.O. Andersson and J. Braun, A neutron rem counter with uniform sensitivity from $0.025 \mathrm{eV}$ to $10 \mathrm{MeV}$, Proc. of the IAEA Symposium on Neutron Detection, Dosimetry and Standardization, Harwell, England, Dec. 10-14, 1962, (IAEA:Vienna) Neutron Dosimetry Vol. II (1963), pp.87-95.

[3] C. Birattari, A. Ferrari, C. Nuccetelli, M. Pelliccioni and M. Silari, An extended range neutron rem counter, Nucl. Instrum. Methods Phys. Res. A297 (1990), pp. 250-257.

[4] L. Jianping, T. Yueli, L. Shudong, S. Ban, T. 
Suzuki, K. Iijima and H. Nakamura, Neutron energy response of a modified Andersson-Braun rem counter, Radiat. Protec. Dosim. 67 (1996), pp. 179-185.

[5] R. H. Olsher, H.-H. Hsu, A. Beverding, J. H. Kleck, W. H. Casson, D. G. Vasilik and R. T. Devine, WENDI: An improved neutron rem meter, Health Physics 79(2) (2000), pp. 170-181.

[6] Y. Nakane, Y. Harada, Y. Sakamoto, T. Oguri, M. Yoshizawa, F. Takahashi, T. Ishikura, T. Fujimoto, S. Tanaka and N. Sasamoto, Evaluation of Energy Response of Neutron Rem Monitor Applied to High-energy Accelerator Facilities, JAERI-Tech 2003-011, Japan Atomic Energy Research Institute, (2003). [in Japanese]

[7] H. Sakai, H. Okamura, H. Otsu, T. Wakasa, S. Ishida, N. Sakamoto, T. Uesaka, Y. Satou, S. Fujita and K. Hatanaka, Facility for the (p,n) polarization transfer measurement, Nucl. Instrum. Methods Phys. Res. A369 (1996), pp. 120-134.

[8] Y. Iwamoto, M. Hagiwara, D. Satoh, H. Iwase, H. Yashima, T. Itoga, T. Sato, Y. Nakane, H. Nakashima, Y. Sakamoto, T. Matsumoto, A. Masuda, J. Nishiyama, A. Tamii, K. Hatanaka, C. Theis, E. Feldbaumer, L. Jaegerhofer, C. Pioch, V. Mares and T. Nakamura, Quasi-monoenergetic neutron energy spectra for 246 and $389 \mathrm{MeV}$ ${ }^{7} \mathrm{Li}(\mathrm{p}, \mathrm{n})$ reactions at angles from 0 to 30 degree, Nucl. Instrum. Methods Phys. Res. A629 (2011), pp. 43-49.

[9] Y. Iwamoto, M. Hagiwara, H. Iwase, H. Yashima, D. Satoh, T. Matsumoto, A. Masuda, C. Pioch, V. Mares, T. Shima, A. Tamii and K. Hatanaka,
Characterization of quasi-monoenergetic neutron source using 137, 200, 246 and $389 \mathrm{MeV}{ }^{7} \mathrm{Li}(\mathrm{p}, \mathrm{n})$ reactions, Proc. ICRS-12\&RPSD-2012, Nara, Japan, Sept. 2-7 (2012) [to be published].

[10]J.F. Briesmeister (Editor), MCNP - A General Monte Carlo n-Particle Transport Code, Version 4B, LA-12625-M, Los Alamos National Laboratory, (1997).

[11]M.B. Chadwick, P.G. Young, S. Chiba, S.C. Frankle, G.M. Hale, H.G. Hughes, A.J. Koning, R.C. Little, R.E. MacFarlane, R.E. Prael and L.S. Waters, Cross section evaluations to $150 \mathrm{MeV}$ for accelerator-driven systems and implementation in MCNPX, Nucl. Sci. Eng. 131(3) (1999), pp. 293-328.

[12]A. Ferrari, M. Pelliccioni and M. Pillon, Fluence to effective dose conversion coefficients for neutrons up to $10 \mathrm{TeV}$, Radiat. Protec. Dosim. 71(3) (1997), pp. 165-173.

[13]A. Ferrari and M. Pelliccioni, Fluence to dose equivalent conversion data and effective quality factors for high energy neutrons, Radiat. Protec. Dosim. 76(4) (1998), pp. 215-224.

[14]T. Sato, A. Endo, M. Zankl, N. Petoussi-Henss, H. Yasuda and K. Niita, Fluence-to-dose conversion coefficients for aircrew dosimetry based on the new ICRP Recommendations, Progress Nucl. Sci. Technol. 1 (2011), pp. 134-137.

[15]Radiation Dose Conversion Coefficients for Radiation Shielding Calculations: 2004, AESJ-SC-R002:2004, Atomic Energy Society of Japan, (2004). [in Japanese] 